\title{
Medicated Film Dosage Form
}

National Cancer Institute

\section{Source}

National Cancer Institute. Medicated Film Dosage Form. NCI Thesaurus. Code C68958.

A film composed of active and/or inert ingredient(s). 